\title{
Hybrid Operations in Patients with Peripheral Arterial Disease
}

\author{
Atsubumi Murakami, MD, PhD
}

In this seminar, I would like to discuss the recent hybrid operations in patients with peripheral arterial diseases. Hybrid is generally defined as combinations of different types of things. In the surgical community, it is loosely defined as therapy combining open surgery (OS) and endovascular therapy (EVT). In practice, combination surgery of diseased inflow vessels by EVT and outflow vessels by OS is a typical example, namely, the combination therapy of thromboendarterectomy (TEA) for common femoral artery and EVT (PTA and stenting) for iliac artery in patients with PAD (ilio-femoral lesions). Also, there is the potential of various combinations of OS and EVT for complex lesions. Unfortunately, we do not have specific guidelines for hybrid therapy of PAD, but in clinical practices, justified decision-making for surgical indication is strictly required. I emphasize that the cardiovascular surgeon (or vascular specialist) must have the ability of decision-making for suitable combination therapy of OS and EVT which adheres to existing specific guidelines. (This is a translation of Jpn J Vasc Surg 2017; 26: 275-283.)

Keywords: hybrid operations, peripheral artery disease (PAD), open surgery, endovascular therapy (EVT), thromboendarterectomy (TEA)

\section{Introduction}

Despite being a common term, the precise definition of

Vascular Surgery, Cardiovascular Center, International University of Health and Welfare Hospital, Nasushiobara, Tochigi, Japan

Received: January 12, 2018; Accepted: January 12, 2018 Corresponding author: Atsubumi Murakami, MD, PhD. Vascular Surgery, Cardiovascular Center, International University of Health and Welfare Hospital, 537-3 Iguchi, Nasushiobara, 329-2763 Tochigi, Japan

Tel: +81-287-39-3060, Fax: +81-287-39-3001

E-mail: atsubumi@iuhw.ac.jp

This is a translation of Jpn J Vasc Surg 2017; 26: 275-283.

(cc) BY-NC-SA (C2018 The Editorial Committee of Annals of Vascular Diseases. This article is distributed under the terms of the Creative Commons Attribution License, which permits use, distribution, and reproduction in any medium, provided the credit of the original work, a link to the license, and indication of any change are properly given, and the original work is not used for commercial purposes. Remixed or transformed contributions must be distributed under the same license as the original. hybrid surgery remains unclear. The term "hybrid" is defined as "something that results from the combination of two different elements" and is considered the etymology underlying the creation of breeds such as inobuta, which is a hybrid between a wild boar (inoshishi) and a domestic pig (buta). At present, it is a term used in many fields such as its use in "hybrid cars." In the field of surgery, what is referred to as endovascular therapy (EVT) is often thought to indicate combination therapy involving open surgery and endovascular therapy (surgery) (EVT (S)). Here, I describe hybrid surgery currently performed in the treatment of peripheral artery disease (PAD).

\section{Suitable Treatment Method Selection for Patients}

The recent success of EVT has significantly altered the therapeutic approach and mindset of surgeons. However, at present, no guidelines exist on the extent to which the two methods can be combined, which would serve as a guide for the most efficient and suitable treatment method. Thus, surgeons today bear the responsibility of creating such guidelines. Hence, while physicians specializing in the treatment of vascular diseases are required to be proficient with the two methods, it would be best to say that vascular surgeons are closest to meeting this requirement.

Treatment options for PAD include pharmacotherapy, exercise therapy, bypass surgery, and EVT; however, the validity of these treatment options is controversial. Recent advancements in EVT techniques and devices have led to day-to-day changes in clinicians' choice as well as the validity of the treatments. To select the most suitable treatment method for patients, it is imperative that the choices are made by the respective guidelines for EVT and open surgery and that the appropriate and most suitable combination is investigated while considering patients' background factors.

Surgeons who treat PAD should remember that they are in a unique position to acquire knowledge about the advantages and disadvantages of both EVT and open surgery, and it is essential that they familiarize with the idea of hybrid surgery, maximizing the advantage of minimally invasive EVT. 


\section{Advantages and Disadvantages of Hybrid Treatment}

Hybrid surgery facilitates the treatment that cannot be achieved by either one when performed alone. Furthermore, it significantly enlarges the scope of treatment and should be maximally utilized. Precisely, it is expected to 1) reduce blood loss, 2) shorten the operative duration, and 3) reduce physical stress, which results in 4) a further reduction in the overall risk of surgery with improved surgical outcomes, and 5) shorter hospital stays. Hence, from the perspective of medical economics and safety, hybrid surgery can be advantageous. In contrast, hybrid surgery requires hybrid operating rooms and investment of the associated equipment. Furthermore, in the absence of guidelines, it is highly likely that the overall outcomes of hybrid surgery depend on the skills of each physician. At present, the long-term outcomes of hybrid surgery are unclear when combined with EVT of the superficial femoral artery (SFA), thereby necessitating comprehensive research when selecting the technique.

\section{Typical Hybrid Treatment in PAD}

Inflow lesions: EVT; outflow lesions: open surgery

When peripheral artery lesions extend over several sites, proximal lesions (referred to as inflow lesions in this study) are treated with EVT, and peripheral lesions (referred to as outflow lesions in this study) are treated with open surgery. Precisely, EVT is performed to treat lesions of the iliac artery, as the advantages of EVT are many in this case, and is a combination of thromboendarterectomy (TEA) or related methods for lesions of the common femoral artery (CFA).

\section{Iliac artery EVT+TEA of the CFA}

For the iliac artery region, EVT has demonstrated high success rate with excellent safety, and the site exhibits no difference in the patency rate in the Trans-Atlantic InterSociety Consensus Document classifications, with a longterm patency rate mostly established at approximately $80 \%$ in 5 years. ${ }^{1,2}$ Conversely, TEA of the CFA (including SFA inlet lesions and angioplasty of some deep femoral artery lesions) has become an established surgical technique with a 5 -year primary patency rate of $91 \%-96 \% .^{3,4)}$ Thus, the combination of the two treatments is considered the most effective hybrid treatment, which is also stated in the present guidelines. When TEA combined with autologous vein patch angioplasty is performed properly as required, it is primarily an effective technique (Figs. 1 and 2).

At my institution, after performing TEA and patch angioplasty, we placed tobacco sutures on a site separate from the site of angioplasty wherever possible, and then performed EVT. However, the approach site and the pro-

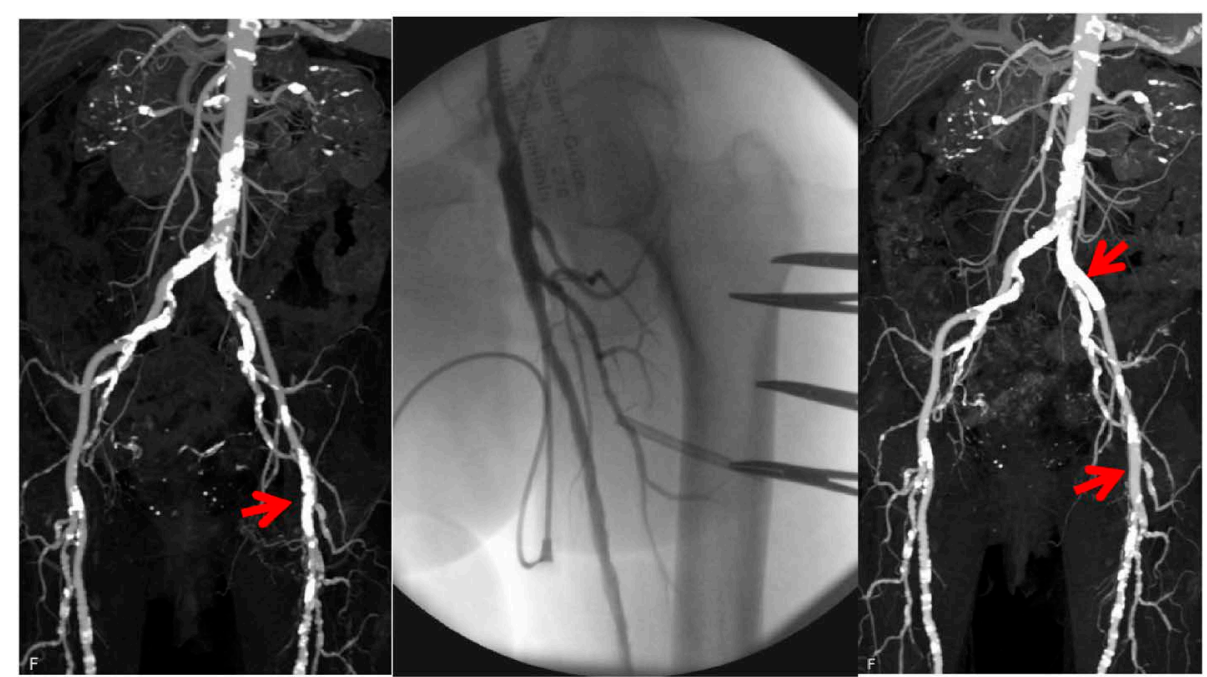

a

$\mathrm{b}$

$\mathrm{C}$

Fig. 1 A case of 72 years old female undergoing HD, post AVR with severe IC of left lower extremity. a: Stenosis with severe calcification of left iliac artery and common femoral artery, preoperative CT (MIP) image. b: Angiography of left common femoral artery (post EVT for iliac artery and TEA with autologous vein patch plasty). Hemostasis of puncture site with ligation of purse string suture. c: Post operative CT (MIP) image. HD: hemodialysis; AVR: aortic valve replacement; IC: intermittent claudication; CT: computed tomography; MIP: maximum intensity projection; EVT: endovascular therapy; TEA: thromboendarterectomy 




a

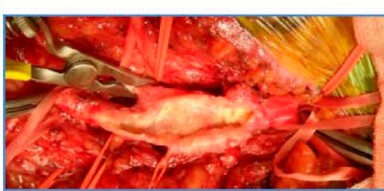

$\mathrm{b}$

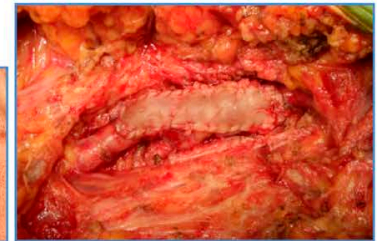

c

Fig. 2 A case of 75 years old female with severe IC (ABI: 0.68). a: Aortotomy. b: Post thromboendarterectomy. c: Post TEA state with autologous vein patch plasty. ABI: anklebrachial pressure index

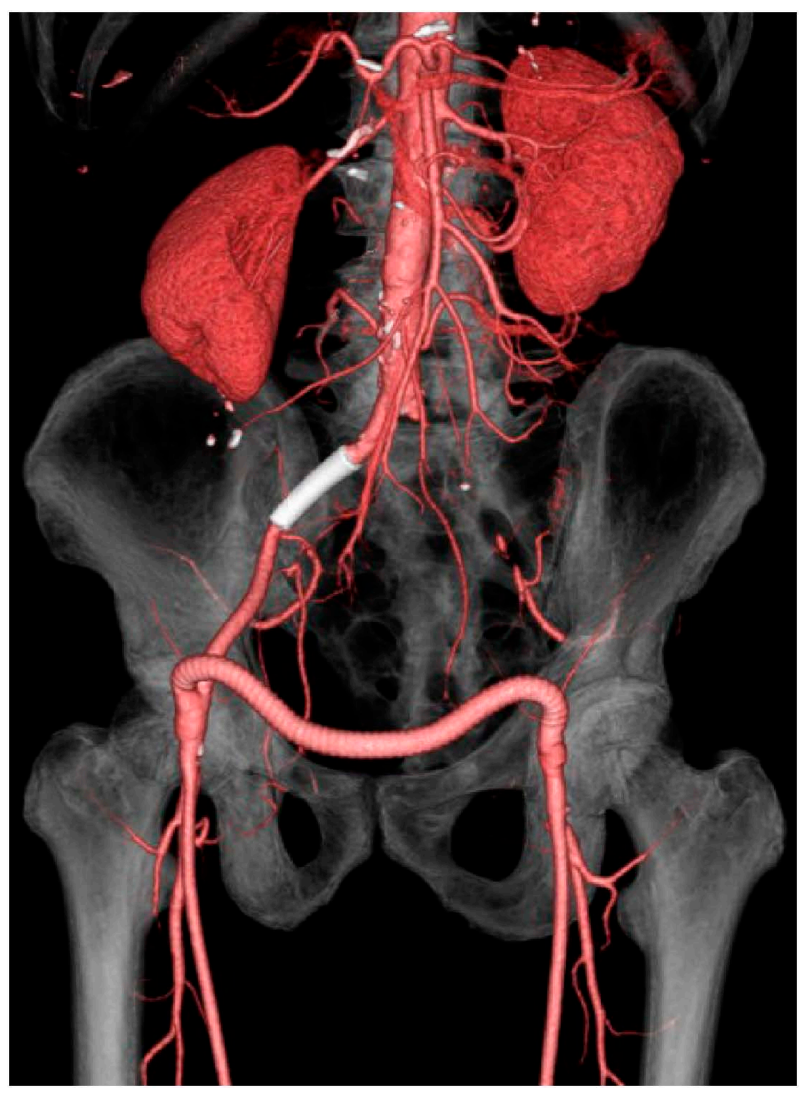

Fig. 3 A case of 63 years old male with severe IC of left lower extremity. Severe stenosis of right iliac artery and obstruction of left iliac artery were identified. Femorofemoral bypass was conducted between right and left common femoral arteries with ring supported $8 \mathrm{~mm}$ ePTFE (FUSION: GETINGE group, Sweden, Gothenburg, Lindholmen) artificial graft after EVT (Wall stent RP $10 \times 20 \mathrm{~mm}$ : Boston Scientific Incorporated, Marlborough, Massachusetts, USA) for right iliac artery. ePTFE: expanded polytetrafluoroethylene

cedure to be performed first can be determined according to the lesion and the practitioner's discretion. Furthermore, in patients with occlusion of the SFA with good arterial patency of the lower leg, the combination with

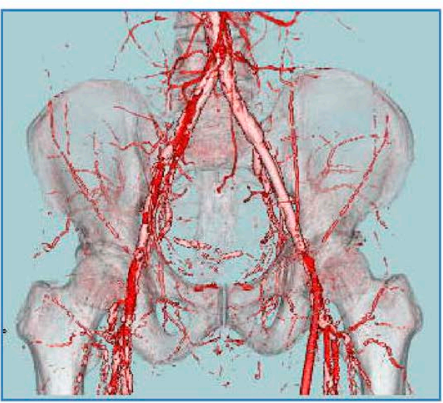

a

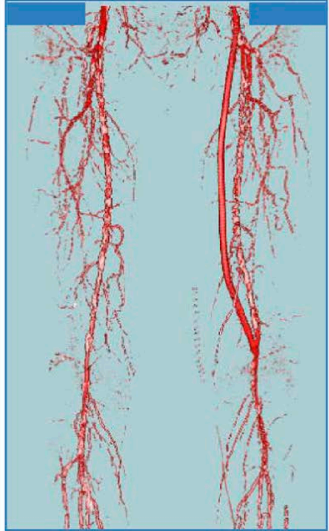

b
Fig. 4 A case of 72 years old male with CLI of left lower extremity. Diffuse severe calcification and severe stenosis of left iliac artery, obstruction of left SFA, diffuse stenosis and obstruction of below knee arteries were identified. a: Post EVT (Wall stent RP $10 \times 39 \mathrm{~mm}$ ) state of left iliac artery. b: Post left femoro-popliteal (above knee) bypass with $6 \mathrm{~mm}$ ePTFE (ADVANTA VXT: GETINGE group, Sweden, Gothenburg, Lindholmen) after TEA of left femoral artery. CLI: critical limb ischemia; SFA: superfidial femoral artery

deep femoral angioplasty alone is considered effective. ${ }^{5,6)}$

Of note, EVT for the CFA has poor outcomes, ${ }^{7}$ and while it is not currently considered a possible alternative to TEA, this might change in future.

Iliac artery EVT combined with other treatments

1) Iliac artery EVT + femoro-femoral crossover (F-F) bypass (Fig. 3)

2) Iliac artery EVT + same side femoropopliteal (F-P) bypass (Fig. 4)

3) Iliac artery EVT + femoral artery TEA + same side F-P bypass (AK, BK)

4) Iliac artery EVT + F-F bypass + contralateral F-P bypass (AK, BK) (Fig. 5)

5) Iliac artery EVT + distal bypass

Combinations such as TEA, F-F bypass, and F-P bypass 
are considered typical methods following EVT for the iliac artery region. The F-F bypass, which is an extra-anatomic bypass, has a slightly inferior 5 -year secondary patency rate of $80 \%-93 \%^{8,9)}$ than that of the anatomic bypass. Furthermore, the autologous vein is recommended for the F-P bypass both above and below the knee; however, in Japan, outcomes with artificial vascular grafts are good



Fig. 5 A case of 81 years old female with severe IC of left lower extremity. Severe stenosis of right iliac artery and obstruction of left iliac artery and SFA were identified. Femorofemoral and femoro-popliteal bypass (sequential) was conducted with ring supported $8 \mathrm{~mm}$ ePTFE (FUSION) artificial graft after EVT (Wall stent RP $10 \times 39 \mathrm{~mm}+$ EPIC $7 \times 60 \mathrm{~mm}$ : Boston Scientific Incorporated, Marlborough, Massachusetts, USA) for right iliac artery. for the bypass AK. ${ }^{10-12)}$ Reportedly, while EVT for the SFA region has a high early success rate and low complication rate, the outcomes for the long-term patency rate, including drug-eluting stents, are not necessarily satisfactory. ${ }^{13,14)}$ However, stent graft outcomes in the SFA region have been good, which might significantly change for this region in the future. ${ }^{15)}$

This procedure varies depending on the state of the patients, the institution, and the practitioner, with the first choice being either the F-P bypass or the F-P bypass after the first choice of EVT for the SFA region, which is unsuccessful. Furthermore, during the application of treatments, the order in which they are performed is critical, and full cooperation is imperative when there are different practitioners for different sites. Based on the present guidelines, ${ }^{1,16-18)}$ further monitoring is required while building evidence. When combining with the distal bypass, the results of the long bypass, as well as the Bypass versus Angioplasty in Severe Ischemia of the Leg (BASIL) trial, should be considered. ${ }^{19,20)}$ Furthermore, these procedures can be indicated for patients with high aortic occlusion.

\section{SFA EVT+BK bypass (Fig. 6)}

In many instances, critical limb ischemia (CLI) is considered a potential disease for the distal bypass with the popliteal artery (POP.A) above the knee (AK) or below the knee (BK) as the inflow in EVT for the SFA. As mentioned earlier, ${ }^{13,14)}$ the present outcomes of EVT for the SFA have poor reliability regarding the inflow. In addition, with
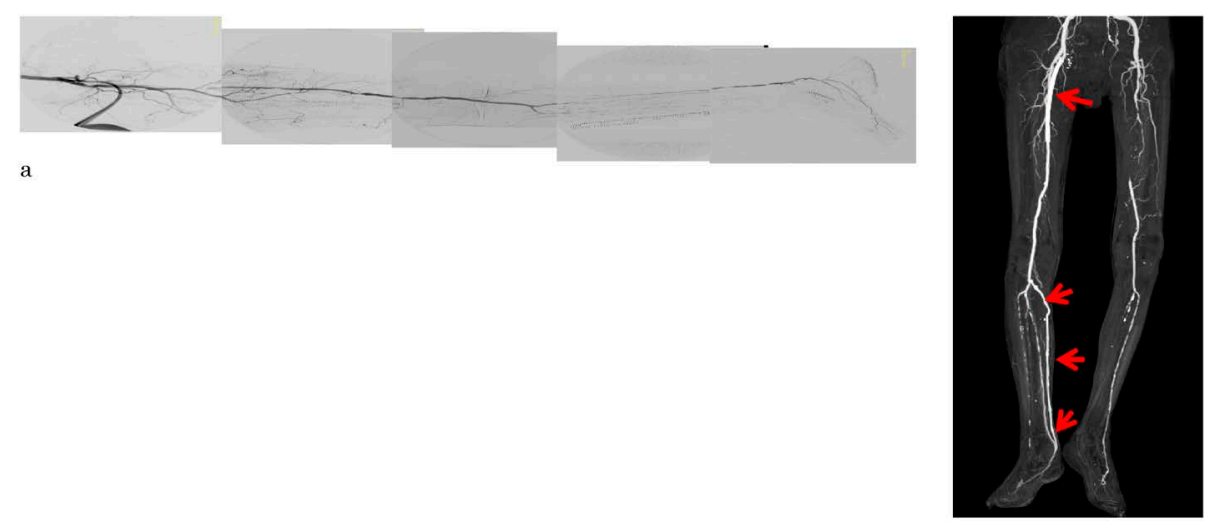

b

Fig. 6 A case of 82 years old male with non-healing sores on the feet. Treatment of ulcer may require surgical procedure. Hybrid procedures were administered, because autologous vein was not favorable for long bypass. a: CT findings, proximal obstruction and stenosis of mid portion of right SFA, diffuse stenosis of below the knee arteries. Arteries on the feet were available for bypass (ABI: 0.28, SPP dorsal portion; $21 \mathrm{mmHg}$, plantar; $15 \mathrm{mmHg}$ ). b: Distal popliteal artery-post tibial artery bypass using non-reversed autologous vein after EVT (Zilver PTX $7 \mathrm{~mm} \times 120 \mathrm{~mm}+7 \mathrm{~mm} \times 60 \mathrm{~mm}$ : Cook Medical Incorporated Bloomington, Indiana, USA) for SFA (Courtesy of Atsuhiro Furuya, Nobuyoshi Azuma, University of Asahikawa). ABI: ankle-brachial pressure index; SPP: skin perfusion pressure 


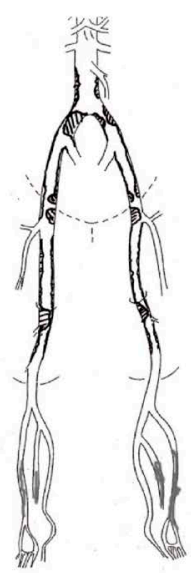

a

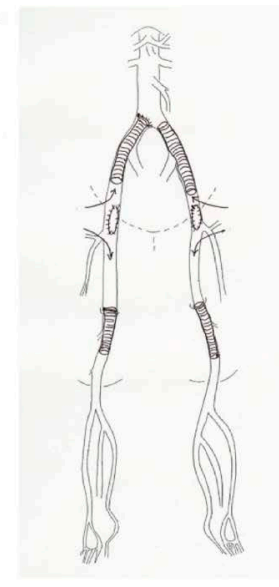

$\mathrm{b}$

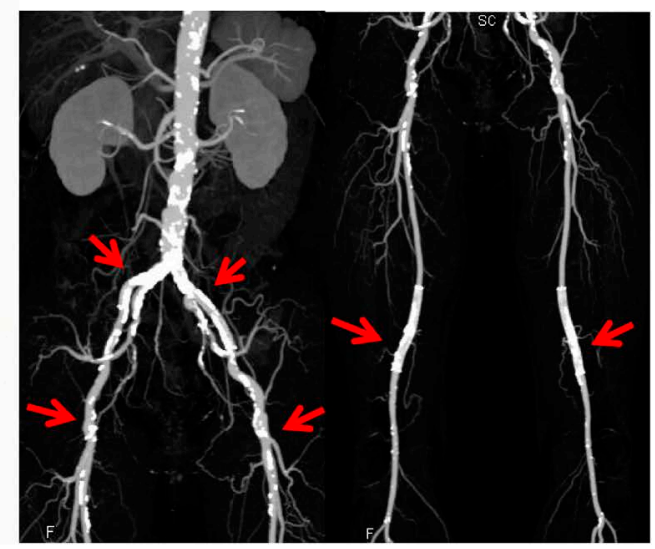

d

Fig. 7 A case of 80 years old male with severe IC of both lower extremity. TEA for both common femoral arteries and EVT were done for both iliac arteries. Simultaneously, EVT for both sides SFA were administered. Postoperative MIP images are indicated (the same case shown in Fig. 9). a: Summary of lesions. b: Final states after hybrid procedures. c: Both sides TEA of common femoral arteries were administered after EVT (Wall stent RP $10 \times 39 \mathrm{~mm}$ ) for right and iliac arteries. d: Simultaneously, EVT for right SFA (Zilver stent $10 \times 60 \mathrm{~mm}$ : Cook Medical Incorporated Bloomington, Indiana, USA) and left SFA (Zilver stent $10 \times 80 \mathrm{~mm}$ ) were administerd.

the problem of the vein graft length that can be primarily used, I believe that there are few cases because this procedure can be chosen (Fig. 6, which is a valuable image provided by Prof. Atsuhiro Furuya and Prof. Nobuyoshi Azuma of Asahikawa Medical University).

\section{Inflow lesions: open surgery; outflow lesions: EVT}

Aorto-iliac (femoral) artery (bilateral, unilateral: aortoiliac, femoral) bypass+SFA, BK EVT

The aorto-iliac (femoral) artery bypass has demonstrated excellent outcomes. ${ }^{21)}$ Accordingly, it is considered in the absence of indications for EVT; however, at present, it is challenging to indicate surgery for several patients without indications for EVT, highlighting the need for careful examination of the indication. When the peripheral anastomosis site is the femoral artery, it is better to perform SFA EVT first, thereby determining the indication and order of the procedures. Of note, the combination of the axillo-femoral artery bypass can also be considered. EVT for BK arteries is, in principle, limited to CLI cases. However, following the BASIL trial,20) indications have extended, and the European Society of Cardiology (ESC) guidelines ${ }^{1)}$ recommend EVT for arteries below the POP.A. In my opinion, the procedure should be considered on the basis of the 2-year survival prediction and the presence or absence of a vein graft.
Femoral artery TEA+SFA, BK EVT (Fig. 7)

Assumedly, the indications for performing this as a onestage procedure will expand.

\section{AK, BK bypass+BK EVT (Fig. 8)}

Following the bypass AK or BK of the POP.A, EVT for BK can be performed; however, such cases are few.

\section{Other}

In the future, the evolution of more diverse combination surgeries is anticipated. At the time of thrombectomy for acute arterial occlusion, simultaneously performing EVT for arteriosclerosis obliterans has become a common practice in recent years.

\section{Essential Precautions When Performing Hybrid Treatment}

1. When performing EVT of the iliac artery, it is imperative to leave the peripheral end of the stent proximal to the iliac circumflex artery from the perspective of the subsequent arterial clamp site. Placing the stent in the CFA must be avoided at all costs. In particular, when cooperating with other departments, I believe that surgeons should remember sharing the fact that surgically performed TEA of the CFA and patch angioplasty are easily performed and have superior long-term patency (Fig. 9).

2. While the long-term patency of EVT for SFA and 



$\mathrm{b}$

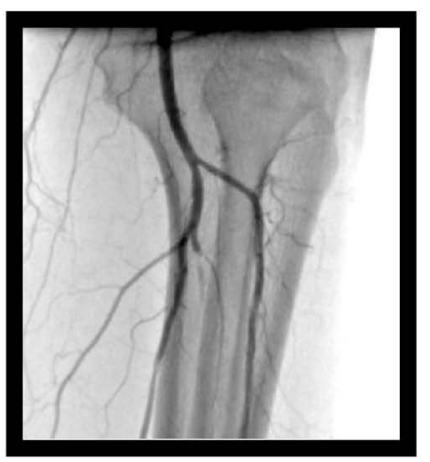

$\mathrm{c}$

a

Fig. 8 A case of 75 years old male with left lower rest pain. a: Post femoro-popliteal (above knee) bypass by $6 \mathrm{~mm}$ ePTFE (Ring supported graft: W. L. Gore \& Associates, Newark, Delaware, USA) artificial graft. b: Preoperative angiography of below the knee arteries. c: EVT for left below the knee arteries through the F-P bypass by ePTFE graft (right femoral artery approach).

BK lesions is poor, patients present polyvascular disease, ${ }^{22}$ and, thus, several patients with PAD are diagnosed with severe coronary artery disease and cerebrovascular disease, which leads to contraindicating open surgery under general anesthesia in several patients. Reportedly, such contraindication is particularly remarkable in dialysis patients with severe calcification and the first choice of treatment is EVT. ${ }^{23)}$

3. Trials of stent grafting for the SFA are completed, and a post-marketing survey is under way in Japan. Although only 1 year has passed, excellent results have been reported, ${ }^{15)}$ and together with further developments in other devices, significant variations in treatment policies are anticipated, which should be closely monitored when considering hybrid treatment.



Fig. 9 (the same case shown in Fig. 7) A case of 80 years old male with severe IC of lower extremity. Severe stenosis were recognized in bilateral iliac, common femoral, superficial femoral arteries with severe calcification. MIP image of right external iliac and common femoral arteries. From the top, arrows indicate proximal puncture site (for iliac PTA), proximal clamp site, TEA site, distal clamp site, distal puncture site (for SFA PTA), respectively. According to anatomy, hybrid operation (shown in Fig. 7) was conducted.

\section{Other Hybrid Treatment Cases}

\section{A case of hybrid surgery for high aortic occlusion (Leriche syndrome)}

[Case]

The case subject was an 87-year-old male with hypertension, atrial fibrillation, cerebral infarction sequelae, and gastric fistula. Although the patient was bedridden, ulcers on his feet could not be healed, which necessitated surgery. 


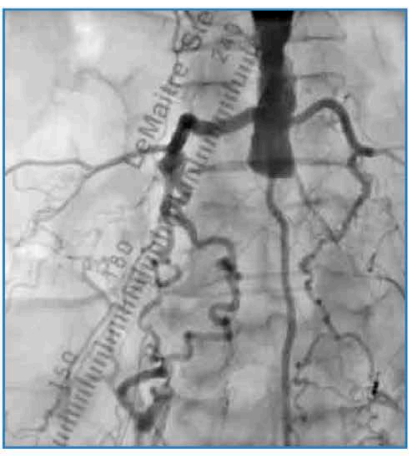

a

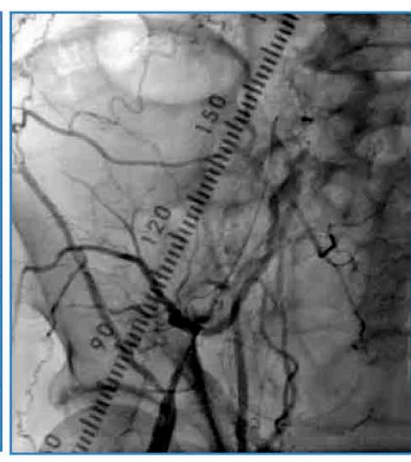

$\mathrm{b}$

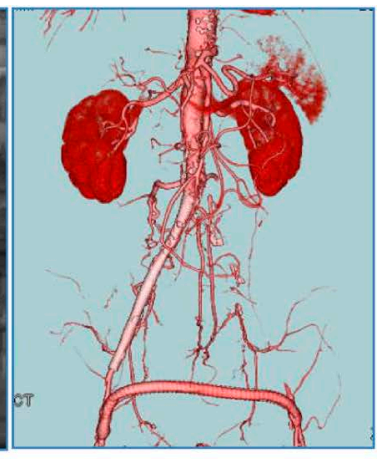

c

Fig. 10 A case of 87 years old male with sores of left feet. a: Aortic occlusion just below the renal arteries. b: EVT for right iliac artery. c: CT image after femoro-femoral bypass using ePTFE graft.

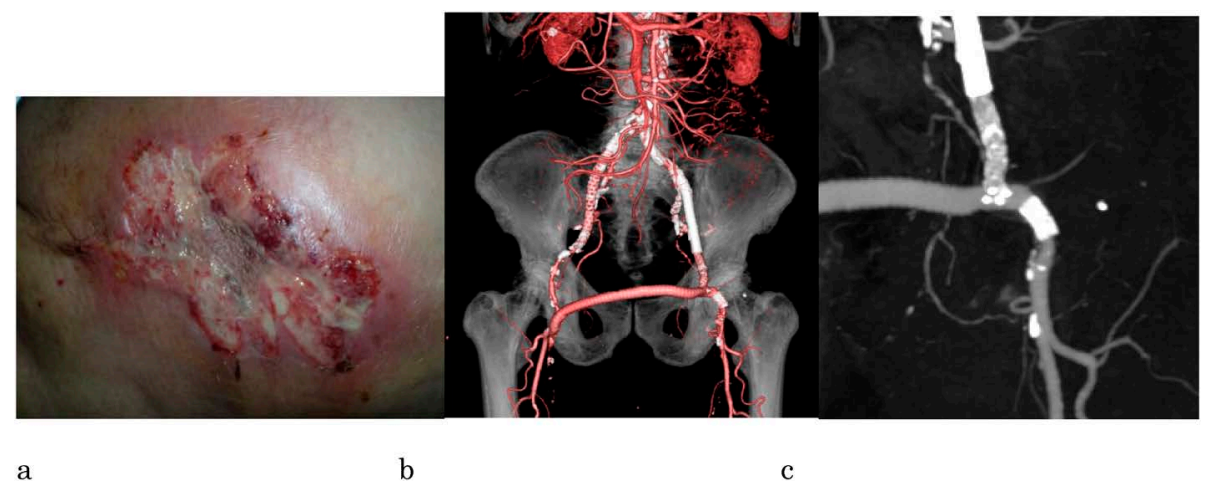

Fig. 11 A case of 84 years old male with rest pain of both lower extremities. a: The skin condition with subcutaneous abscess formation. b: Stenting (Zilver stent $5187 \times 60 \mathrm{~mm}$ : Cook Medical Incorporated, Bloomington, Indiana, USA) between stent in the external iliac artery and artificial graft. c: CT (MIP image) demonstrated stents connected to artificial graft.

\section{[Treatment progress]}

A. Iliac artery EVT (Wall stent RP $10 \times 39 \mathrm{~mm}$, Boston Scientific Incorporated, Marlborough, Massachusetts, USA)

B. The right F-F crossover bypass (ring support $8 \mathrm{~mm}$ ePTFE graft; W. L. Gore \& Associates Inc., Newark, Delaware, USA) (Fig. 10)

[Summary]

Several recent reports have described bilateral EVT alone for high aortic occlusion. In this case, as EVT was commenced early, hybrid treatment was planned from the beginning. The decision to perform Y graft, hybrid treatment, or EVT alone should be determined after comprehensive assessment of all indications.

\section{A case in which the leg was successfully saved by EVT for an infection of the site of artificial vascular graft anastomosis in the femoral artery following a hybrid surgery for CLI [Case]}

The case subject was an 84-year-old male with CLI accompanied by angina (following percutaneous coronary intervention), chronic renal failure (hemodialysis (HD)), and sinus node dysfunction (pacemaker (PM)). The patient presented right iliac artery occlusion (following EVT at a different hospital), 99\% stenosis of the left iliac artery, $90 \%$ stenosis of the right femoral artery, occlusion of the bilateral SFA, and below the knee triple vessels with stenosis and/or occlusion.

[Treatment progress]

A. The subject underwent left iliac artery EVT (Wall stent RP $10 \times 30 \mathrm{~mm}$ ).

B. The right deep F-F crossover bypass (ring support $8 \mathrm{~mm}$ ePTFE graft). Removal of hematoma was per- 
formed for postoperative bleeding in the left femur; however, wound infection developed, and although the infection could be controlled by conservative treatment, the left femoral artery stenosis and deep femoral artery stenosis progressed (Fig. 11a).

Zilver stent $5187 \mathrm{~mm} \times 60 \mathrm{~mm}$ (Cook Medical Incorporated, Bloomington, Indiana, USA) was placed extending the left iliac artery stent and artificial graft, and an Express SD $7 \mathrm{~mm} \times 14 \mathrm{~mm}$ (Boston Scientific Incorporated, Marlborough, Massachusetts, USA) was placed in the left deep femoral artery (Figs. 11b and 11c).

[Summary]

After treatment, the patient became ambulatory and has been leading a stable life for $>5$ years. This case suggests that when the indication of open surgery is difficult on the basis of infection and the patient's general condition, the CFA can be exceptionally treated by EVT.

\section{Hybrid Operating Rooms}

With recent developments witnessed in hybrid operating rooms, their utilization has also expanded, and its various applications are listed below.

The applications include endoluminal grafting (TEVAR, EVAR), combined surgery such as with percutaneous transluminal angioplasty for lower leg vessels and bypass, pacemaker placement, transcatheter aortic valve implantation, transcatheter mitral valve repair, transcatheter pulmonary valve replacement, catheter embolization for atrial septal defects and oval foramen, left atrial appendage closure, transcatheter arterial duct closure, hybrid treatment for hypoplastic left heart syndrome, craniotomy + angiography, and craniotomy + endovascular treatment. Furthermore, the utilization is not limited to hybrid surgery and operative rooms are being developed with excellent mobility to help improve the rate of utilization.

\section{Conclusion}

I described the indications and types of hybrid surgery as well as hybrid operating rooms in the field of PAD treatment.

\section{Disclosure Statement}

The author has no conflict of interest to declare regarding this article.

\section{Additional Remarks}

This article was announced at the 45th annual meeting of the Japanese Society for Vascular Surgery (April 2017, Hiroshima), and the 25th educational seminar for the
Japanese Society for Vascular Surgery.

\section{References}

1) Tendera M, Aboyans V, Bartelink ML, et al. ESC Guidelines on the diagnosis and treatment of peripheral artery disease: document covering atherosclerotic disease of extracranial carotid and vertebral, mesenteric, renal, upper and lower extremity arteries: the Task Force on the Diagnosis and Treatment of Peripheral Artery Diseases of the European Society of Cardiology (ESC). Eur Heart J 2011; 32: 2851906.

2) Kashyap VS, Pavkov ML, Bena JF, et al. The management of severe aortoiliac occlusive disease: endovascular therapy rivals open reconstruction. J Vasc Surg 2008; 48: 1451-7.e3.

3) Ballotta E, Gruppo M, Mazzalai F, et al. Common femoral artery endarterectomy for occlusive disease: an 8-year singlecenter prospective study. Surgery 2010; 147: 268-74.

4) Kang JL, Patel VI, Conrad MF, et al. Common femoral artery occlusive disease: contemporary results following surgical endarterectomy. J Vasc Surg 2008; 48: 872-7.

5) Kalman PG, Johnston KW, Walker PM. The current role of isolated profundaplasty. J Cardiovasc Surg (Torino) 1990; 31: 107-11.

6) Koscielny A, Pütz U, Willinek W, et al. Case-control comparison of profundaplasty and femoropopliteal supragenicular bypass for peripheral arterial disease. Br J Surg 2010; 97 : 344-8.

7) Bonvini RF, Rastan A, Sixt S, et al. Endovascular treatment of common femoral artery disease: medium-term outcomes of 360 consecutive procedure. J Am Coll Cardiol 2011; 58: 792-8.

8) Ricco JB. Unilateral iliac artery occlusive disease: a randomized multicenter trial examining direct revascularization versus crossover bypass. Ann Vasc Surg 1992; 6: 209-19.

9) Mingoli A, Sapienza P, Feldhaus RJ, et al. Comparison of femorofemoral and aortofemoral bypass for aortoiliac occlusive disease. J Cardiovasc Surg (Torino) 2001; 42: 381-7.

10) Johnson WC, Lee KK. A comparative evaluation of polytetrafluoroethylene, umbilical vein, and saphenous vein bypass grafts for femoral-popliteal above-knee revascularization: a prospective randomized Department of Veterans Affairs cooperative study. J Vasc Surg 2000; 32: 268-77.

11) Inoue $Y$, Sugano N, Jibiki M, et al. Cuffed anastomosis for above-knee femoropopliteal bypass with a stretch expanded polytetrafluoroethylene graft. Surg Today 2008; 38: 679-84.

12) Pereira CE, Albers M, Romiti $M$, et al. Meta-analysis of femoropopliteal bypass grafts for lower extremity arterial insufficiency. J Vasc Surg 2006; 44: 510-7.

13) Soga Y, Iida O, Hirano K, et al. Mid-term clinical outcome and predictors of vessel patency after femoropopliteal stenting with self-expandable nitinol stent. J Vasc Surg 2010; 52: 608-15.

14) Dake MD, Ansel GM, Jaff MR, et al. Paclitaxel-eluting stents show superiority to balloon angioplasty and bare metal stents in femoro-popliteal disease: twelve-month Zilver PTX randomized study results. Circ Cardiovasc Interv 2011; 4 : 495-504.

15) Ohki T, Kichikawa K, Yokoi H, et al. Outcomes of the Japanese multicenter Viabahn trial of endovascular stent 
grafting for superficial femoral artery lesions. J Vasc Surg 2017; 66: 130-42.e1.

16) Anderson JL, Halperin JL, Albert NM, et al. Management of patients with peripheral artery disease (compilation of 2005 and 2011 ACCF/AHA guideline recommendations): a report of the American College of Cardiology Foundation/ American Heart Association Task Force on Practice Guidelines. Circulation 2013; 127: 1425-43.

17) Conte MS, Pomposelli FB, Clair DG, et al. Society for Vascular Surgery practice guidelines for atherosclerotic occlusive disease of the lower extremities: management of asymptomatic disease and claudication. J Vasc Surg 2015; 61 Suppl: 2S-41S.e1.

18) Guidelines for the management of peripheral arterial occlusive diseases (JCS 2015).

19) Shah DM, Darling RC 3rd, Chang BB, et al. Is long vein bypass from groin to ankle a durable procedure? An analysis of a ten-year experience. J Vasc Surg 1992; 15: 402-7; discussion, 407-8.
20) Bradbury AW, Adam DJ, Bell J, et al. Bypass versus Angioplasty in Severe Ischemia of the Leg (BASIL) trial: an intention-to-treat analysis of amputation-free and overall survival in patients randomized to a bypass surgery-first or a balloon angioplasty-first revascularization strategy. J Vasc Surg 2010; 51 Suppl: 5S-17S.

21) de Vries SO, Hunink MG. Results of aortic bifurcation grafts for aortoiliac occlusive disease: a meta-analysis. J Vasc Surg 1997; 26: 558-69.

22) Ohman EM, Bhatt DL, Steg PG, et al. The Reduction of Atherothrombosis for Continued Health (REACH) Registry: an international, prospective, observational investigation in subjects at risk for atherothrombotic events-study design. Am Heart J 2006; 151: 786.e1-10.

23) Meyer A, Lang W, Borowski M, et al. In-hospital outcomes in patients with critical limb ischemia and end-stage renal disease after revascularization. J Vasc Surg 2016; 63: 96673. 